\title{
A comparison of the magnetic properties of Proton- and Iron-implanted graphite
}

\author{
J. Barzola-Quiquia, R. Höhne, M. Rothermel, A. Setzer, and P. Esquinaz* \\ Institut für Experimentelle Physik II, Universität Leipzig, Linnéstraße 5, D-04103 Leipzig, Germany \\ V. Heera \\ Institute of Ion Beam Physics and Materials Research, \\ Forschungszentrum Dresden-Rossendorf, P.O.Box 510119, D-01314 Dresden, Germany
}

\begin{abstract}
In this work we have investigated the changes of the magnetic properties of highly oriented pyrolytic graphite samples after irradiation either with $\sim 3 \times 10^{14}$ protons or $3.5 \times 10^{13} \ldots 3.5 \times 10^{14}$ iron ions with energies in the $\mathrm{MeV}$ range. Our results show that iron and proton irradiations can produce similar paramagnetic contributions depending on the implantation temperature. However, only protons induce a ferromagnetic effect.

PACS numbers: 75.50.Pp,78.70.-g,81.05.Uw
\end{abstract}

The possible existence of ferromagnetic order in metalfree carbon attracted some interest of basic and material research scientists since the end of the 60 's $1,1,2,3,3,4$. However, only in the last years and partially due to the technical improvement in the magnetic detection as well as in the analytical methods to determine the impurity contribution to the magnetic properties, this subject shows nowadays a renaissance. Theoretical studies showed that a mixture of $\mathrm{sp}^{2}$-sp ${ }^{3}$-bonded carbon atoms with unpaired $\pi$-electrons [5] or different numbers of mono- and dihydrogenated carbon atoms [6], both within a graphite-like structure, may trigger magnetic order. New theoretical approaches suggest, however, that lattice defects and/or the influence of atomic hydrogen coupled to a carbon atom of the graphene lattice might induce a spontaneous magnetization [7, 8, 9, 10]. Depending on the density and position of the defects, different Curie temperatures are expected. The influence of an hydrogen-like particle on the magnetic response of the graphite lattice has been indirectly observed by muon spin resonance ( $\mu \mathrm{SR})$ experiments on highly oriented pyrolytic graphite (HOPG) [11], which reveal the formation of a magnetic moment in the surrounding of the muon. It is important to note that in the theoretical approaches the graphite/graphene lattice plays a main role, i.e. strictly speaking amorphous carbon or a highly disordered graphite lattice is not expected to reveal spontaneous magnetization. Whether the ferromagnetic carbon films reported in Refs. 12, 13, 14 were highly disordered or amorphous remains still unclear since apparently nobody could reproduce those results yet.

Whereas earlier obtained experimental evidence for room temperature magnetic order in graphite 15, 16, 17, 18] has been recently confirmed [19, 20, 21], part of the scientific community still doubts concerning the intrinsic character of the observed effects added to a special role of iron in a carbon matrix [22, 23]. The aim of the present study was to compare the magnetic properties of HOPG samples bombarded by a similar number of protons- and iron-ions at similar energies. Our exper- imental work shows that iron bombardment under the used irradiation conditions and up to a concentration of $265 \mathrm{ppm}$ (in the irradiated region) does not trigger magnetic order in graphite, in clear contrast to proton irradiation.

In these studies two main improvements were realized in comparison with earlier reports on the measurements of the magnetic properties of proton irradiation of HOPG samples [17, 18]. Firstly, in order to reduce the effective thermal annealing of the defects produced during irradiation an special chamber was developed that enables us to cool down the sample at a nominal temperature of $110 \mathrm{~K}$ during irradiation. Second, a special sample holder was designed that allows us to measure the sample in the SQUID and to fix it inside the irradiation chamber without any changes [20]. This last improvement provides a reproducibility in magnetic moment of $\sim 10^{-7}$ emu. In the case of iron implantation the samples must be every time fixed on the holder for SQUID measurements after irradiation. Therefore, small deviations exist in the sample orientation respect to the applied magnetic field between measurements performed before and after irradiation, which influences mainly the measured diamagnetic contribution. This contribution should be accordingly subtracted in order to obtain any para- and/or ferromagnetic contributions.

For the present investigations HOPG samples of grade ZYA $\left(0.4^{\circ}\right.$ mosaicity) were used. To trigger ferromagnetism by proton irradiation of HOPG broad- as well as micro-beam technique can be used 21. The sample HG1 was irradiated at $110 \mathrm{~K}$ with a $2.25 \mathrm{MeV}$ proton microbeam perpendicular to the graphite planes. The irradiated sample is called HG1_H (the same sample before irradiation is called HG1_V). The irradiation was performed on $160 \times 160$ spots of $\simeq 2.6 \mu \mathrm{m}$ radius each and separated by a distance of $10 \mu \mathrm{m}$ on a total area of $2.5 \mathrm{~mm}^{2}$. The dose (fluence) and total charge were $5 \times 10^{16} \mathrm{~cm}^{-2}\left(0.08 \mathrm{nC} / \mu \mathrm{m}^{2}\right)$ and $44.8 \mu$ C, i.e. a total of $\simeq 2.8 \times 10^{14}$ protons up to a depth of $\sim 46 \mu \mathrm{m}$ from the surface. The proton current used was $1 \mathrm{nA}$. 


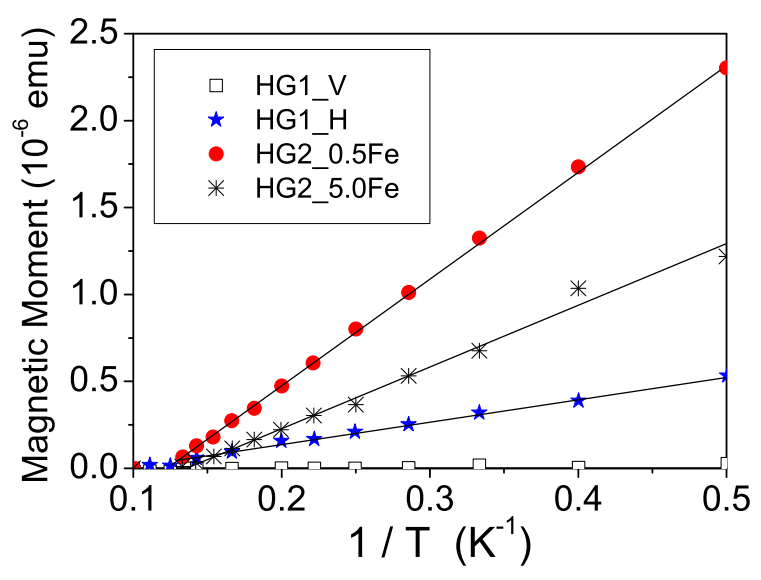

FIG. 1: Magnetic moment in units of $10^{-6} \mathrm{emu}(1 \mathrm{emu} / \mathrm{g}=$ $\left.1 \mathrm{Am}^{2} / \mathrm{kg}\right)$ measured at $H=2 \mathrm{kOe}\left(1 \mathrm{Oe}=10^{3} / 4 \pi \mathrm{A} / \mathrm{m}\right)$ after ZFC from $300 \mathrm{~K}$ to $2 \mathrm{~K}$ as a function of the reciprocal temperature for sample HG1 in the virgin state $(\square)$ and after proton irradiation $(\star)$ as well as for sample HG2 after the first $(\bullet)$ and the second iron implantation $(*)$. The curves are obtained after subtracting the magnetic moment at $T=10 \mathrm{~K}$ and $2 \mathrm{kOe}$. The straight lines are linear fits to the data.

Using particle induced x-ray emission we determined a magnetic impurity content of $0.4 \mu \mathrm{g} / \mathrm{g}$ of $\mathrm{Fe}(<0.1 \mathrm{ppm})$ homogeneously distributed in the sample.

Sample HG2_V was implanted with Fe-ions onto an area of $6.9 \mathrm{~mm}^{2}$ up to a depth of $\simeq 2.1 \mu \mathrm{m}$ by multienergy implantation in the range between $1 \ldots 4.0 \mathrm{MeV}$. The sample was implanted with a fluence of $5 \times$ $10^{14} \mathrm{Fe} / \mathrm{cm}^{2}$ (sample HG2_0.5Fe). After magnetic characterization, the same sample was irradiated at $450^{\circ} \mathrm{C}$ with a fluence of $5 \times 10^{15} \mathrm{Fe} / \mathrm{cm}^{2}$ on the same surface after renewing it by peeling out the earlier irradiated surface; this sample is called HG2_5.0Fe. The achieved Fe concentration in the implanted depth is $26.5 \mathrm{ppm}$ for sample HG2 $20.5 \mathrm{Fe}$ and $265 \mathrm{ppm}$ for sample HG2_5.0Fe. The measurements of the magnetic moment were carried out with a SQUID magnetometer from Quantum Design with a reciprocating sample option (RSO) and a resolution of $\sim 2 \times 10^{-8} \mathrm{emu}$. Both samples HG1 and HG2 were characterized magnetically in the virgin as well as in the irradiated states. The field was applied always parallel to the graphene layers to reduce the diamagnetic contribution to the magnetic moment.

In the investigated temperature range we observe three contributions to the magnetization of the same graphitic phase: a diamagnetic one due to orbital effects, a paramagnetic one caused by the lattice disorder before and (largely enhanced) after irradiation, and a weak ferromagnetic one with a Curie temperature above $300 \mathrm{~K}$. The paramagnetic contribution is visible in an up-turn of the
$T$-dependence of the magnetic moment at $T \lesssim 20 \mathrm{~K}$. Figure 1 shows the magnetic moment $m$ vs. inverse $T$ after subtraction of the value $m(10 \mathrm{~K})$ at the applied field of $2 \mathrm{kOe}$. Due to the practically $T$-independence of the ferromagnetic and diamagnetic contributions between $2 \mathrm{~K}$ and $10 \mathrm{~K}$, this subtraction shows basically the paramagnetic contribution, which follows the $1 / T$ Curie-law dependence, see Fig. 1. The slope of the lines in Fig. 1 is proportional to the density of paramagnetic centers. The paramagnetic contribution of the sample in the virgin state $\left(\mathrm{HG} 1_{-} \mathrm{V}\right)$ is very small whereas the disorder created by the particle bombardment enhances this contribution.

We note that the implantation with iron performed at room temperature shows the highest paramagnetism among all samples. The paramagnetic contribution induced by iron implantation at the same temperature increases with implantation dose. It decreases, however, by increasing the implantation temperature or by a postimplantation heat treatment. The sample $\mathrm{HG} 2_{-} 5.0 \mathrm{Fe}$ was implanted with the tenfold dose of that of sample HG2 $-0.5 \mathrm{Fe}$ but at $T=450{ }^{\circ} \mathrm{C}$. Because of the strong recombination of the carbon vacancies created by the $\mathrm{Fe}$ bombardment the paramagnetic contribution is smaller than that in sample $\mathrm{HG} 2_{-} 0.5 \mathrm{Fe}$ and can be further decreased by a factor of two after a heat treatment at $700{ }^{\circ} \mathrm{C}$ in high vacuum for two hours.

For a quantitative comparison we estimate the number of Bohr magnetons $\mu_{B}$ in the samples using the Curie law and the assumption that each magnetic center contributes with one $\mu_{B}$. From the slopes of the curves in Fig. 1 one gets that the proton irradiation of sample HG1 has induced $3.2 \times 10^{15} \mu_{B}$, the samples HG2_- $0.5 \mathrm{Fe}$ Fe-implanted at room temperature and HG2_5.0Fe Feimplanted at $450{ }^{\circ} \mathrm{C}$ contain $\simeq 14.6 \times 10^{15} \mu_{B}$ and $\simeq 8.1 \times 10^{15} \mu_{B}$, respectively. We note that in the sample HG2_5.0Fe a particular part of the paramagnetic contribution is caused by the paramagnetic Fe-ions themselves. Let us compare these values in relation to the number of implanted particles. From the total charge of $44.8 \mu \mathrm{C}$ and the charge of an electron one gets $2.8 \times 10^{14}$ implanted protons in sample HG1_H. Into the two Fe-implanted samples we implanted $3.45 \times 10^{13}$ and $3.45 \times 10^{14} \mathrm{Fe}-$ ions. From these values follows that the numbers of Bohr magnetons per implanted particle is $11 \pm 2,420 \pm 100$ and $23 \pm 5$ for samples HG1_H, HG2_ 0.5 Fe and HG2_ 5.0 $\mathrm{Fe}$, respectively. The relatively large (maximal) error of these values for the Fe-implanted samples comes from the possible deviations between the nominal and the realized iron implantation dose and the uncertainties concerning the paramagnetic contribution of sample HG2 in its virgin state. Without doubt the maximum disorder is created by the Fe-implantation at room temperature. This in principle correlates with the results of SRIM simulations 24]. The integral of the damage distribution caused by the implantation under the here used 


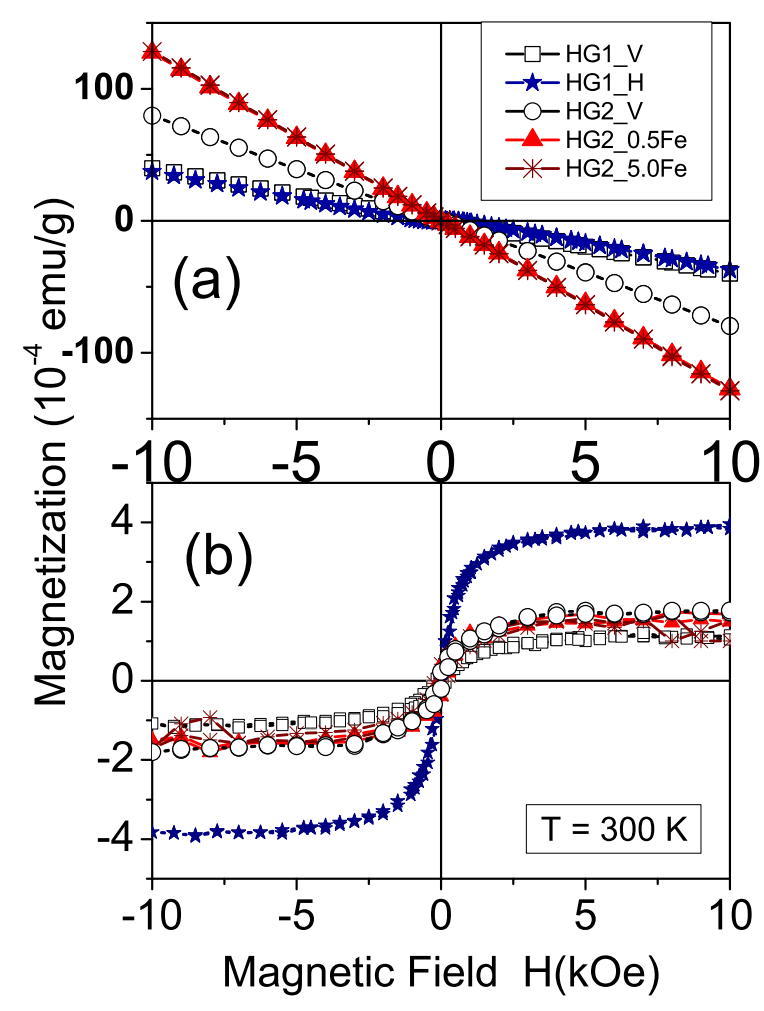

FIG. 2: Magnetization in units of $10^{-4} \mathrm{emu} / \mathrm{g}$ (related to the whole sample mass) measured at $T=300 \mathrm{~K}$ as a function of the magnetic field for the samples before (open symbols) and after irradiation with protons $(\star)$ or Fe-ions $(\boldsymbol{\Lambda}$, sample HG2_ $0.5 \mathrm{Fe}, *$, sample $\mathrm{HG} 2 \_5.0 \mathrm{Fe}$ ) without any subtraction (a) and after subtraction of the linear diamagnetic part (b). The remanent magnetization and coercive fields for sample HG1_V , for example, are $\simeq 2.4 \times 10^{-5} \mathrm{emu} / \mathrm{g}$ and $\simeq 0.16 \mathrm{kOe}$.

conditions gives 15 and 4000 carbon displacements (vacancies) per implanted proton and iron ion. For example, for the sample $\mathrm{HG} 22_{-} 0.5 \mathrm{Fe}$ we get a vacancy concentration of $1 \times 10^{22}$ vacancies $/ \mathrm{cm}^{3}$ in the first $\sim 2 \mathrm{~nm}$ from the surface. Compared with the atomic density of carbon in HOPG this value corresponds to 0.088 displacements per atom. However, most of these vacancies are not stable and due to vacancy-interstitial recombination the damage is reduced by almost one order of magnitude, i.e. instead of $4000 \mu_{B}$ (assuming $1 \mu_{B}$ per vacancy) we obtain experimentally $\simeq 420 \mu_{B}$ per implanted Fe-ion. Nevertheless the lattice disorder remains high in the Feimplanted sample HG2_ $0.5 \mathrm{Fe}$.

For the discussion of the lattice damage we have to take into account the different defect distribution for iron and proton irradiations. Whereas this distribution is rather homogeneous in our Fe-implanted samples (due to the multi-energy implantation method), the $2.25 \mathrm{MeV}$ proton irradiation shows a sharp defect density maximum at $\sim 46 \mu \mathrm{m}$. However, the lattice disorder in the

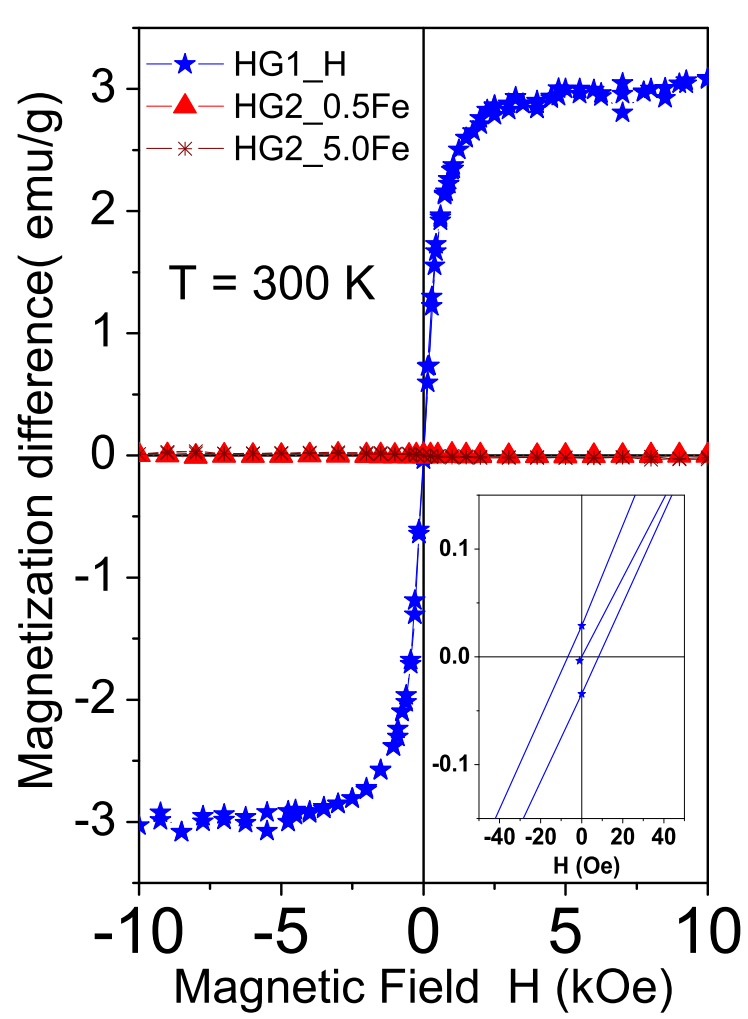

FIG. 3: Magnetization in units of emu/g for the irradiated samples after subtraction of the contribution of the corresponding samples in the virgin state measured at $T=300 \mathrm{~K}$ as a function of the magnetic field. The magnetization is related to the estimated ferromagnetic mass of the irradiated sample region only. The inset shows the region around zero field of the proton irradiated sample HG1_H.

proton-irradiated sample is relatively small in the ferromagnetically active layer, which is localized in the first micrometers 21]. In this region the proton irradiation produced a vacancy density of $\simeq 5 \times 10^{20} \mathrm{~cm}^{-3}$. We note that this estimated density from SRIM simulations is 20 times smaller than the one for the Fe-irradiation of sample HG2 $0.5 \mathrm{Fe}$. The ratio 20 between these densities of vacancies is comparable with the experimental ratio of 40 for the numbers of Bohr magnetons per implanted particle.

The magnetization measured at $T=300 \mathrm{~K}$ by cycling the magnetic field between $\pm 10 \mathrm{kOe}$ is given in Fig. 2(a). For all samples the magnetization curves are almost linear, dominated by the diamagnetism. The differences of the slopes between the curves for the samples HG1 and HG2 and between the HG2 curves are caused primarily by small, different misalignments between the external applied magnetic field and the sample main plane. After subtraction of the dominating linear background small ferromagnetic loops remain, see 
Fig. 2(b). Ferromagnetic-like loops are also seen in the virgin samples [16]. The proton irradiation performed at low temperatures causes a clear enhancement of the ferromagnetic effect in relation to that of the virgin sample. In contrast, the Fe-implantation does not lead to a detectable increase of the ferromagnetism. Even a Feimplantation with a tenfold dose (sample HG2_Fe5.0) gives no enhancement of the ferromagnetic contribution.

The ferromagnetic behaviour of the irradiated samples is better demonstrated in Fig. 3 where the magnetization curves of the samples HG1 and HG2 are plotted after subtraction of the contributions of the corresponding virgin samples. To show the influence of irradiation the magnetization in Fig. 3 is related to the mass of the irradiated sample region. It is known that the ferromagnetically active layer in a proton-irradiated graphite sample is very small in comparison to the penetration depth of the protons for the fluence and energy used here [21]. For the determination of the irradiated area of sample HG1 we assumed a spot radius of $2.6 \mu \mathrm{m}$ and a thickness of the ferromagnetic part of $1 \mu \mathrm{m}$. After subtraction of the paramagnetic part we get a value of $3 \mathrm{emu} / \mathrm{g}$ at $T=300 \mathrm{~K}$ for the ferromagnetic saturation magnetization caused by the proton irradiation. If we assume a larger area due to irradiation broadening, i.e. $2.5 \mathrm{~mm}^{2}$, we estimate a magnetization at saturation of the order of $0.6 \mathrm{emu} / \mathrm{g}$.

Concluding, for similar amount of irradiated ions under similar energies our results show clearly that only the proton-irradiation induces a ferromagnetic contribution under the conditions used in this study. In comparison with proton-irradiation in the Fe-implanted samples the structural disorder, i.e. vacancy density, produced by the bombardment appears to be too large to induce ferromagnetic correlations. A large disorder in the atomic lattice may prevent the existence of a long range magnetic interaction through the graphene structure [10, 25]. We speculate that the ferromagnetism in the protonirradiated sample is a combination of the disorder plus the action of hydrogen ions (already in the sample very probably as $H_{2}$ and dissociated by the irradiation). Independently done electric force measurements and scanning transmission x-ray microscopy on magnetic spots provide further evidence that the irradiation induces a change in the electronic $\pi$-band and $\sigma$-band, leaving the ferromagnetic phase with a smaller conduction electron density at Fermi level [26], a result compatible with recently done theoretical work 25] that indicates that the ferromagnetic graphitic phase should have insulating properties.

We gratefully acknowledge discussions with N. García, M. A. Vozmediano, L. Pisani and N. Harrison and T. Butz for the permanent support. This work was done in the framework of the EU project "Ferrocarbon" and partially supported by the DFG under ES 86/11.
[1] N. Mataga, Theoret. Chim. Acta (Berl.) 10, 372 (1968).

[2] N. Tyutyulkov and I. Bangov, Compt. Rend. Acad. Bulg. Sci. 27, 1517 (1974).

[3] A. A. Ovchinnikov, Theoret. Chim. Acta (Berl.) 47, 297 (1978).

[4] T. Makarova, Semiconductors 38, 615 (2004).

[5] A. A. Ovchinnikov and I. L. Shamovsky, J. of Molecular Structure (Theochem) 251, 133 (1991).

[6] K. Kusakabe and M. Maruyama, Phys. Rev. B 67, 092406 (2003).

[7] J. González, F. Guinea, and M. A. H. Vozmediano, Phys. Rev. B 63, 134421 (2001).

[8] P. O. Lehtinen, A. S. Foster, Y. Ma, A. Krasheninnikov, and R. M. Nieminen, Phys. Rev. Lett. 93, 187202 (2004).

[9] E. J. Duplock, M. Scheffler, and P. J. D. Lindan, Phys. Rev. Lett. 92, 225502 (2004).

[10] O. V. Yazyev and L. Helm, Phys. Rev. B 75, 12548 (2007).

[11] J. A. Chakhalian, R. F. Kiefl, S. R. Dunsiger, W. A. MacFarlane, R. Miller, J. E. Sonier, and J. E. Fischer, Phys. Rev. B 66, 155107 (2002).

[12] K. Murata, H. Ushijima, H. Ueda, and K. Kawaguchi, J. Chem. Soc., Chem. Commun. pp. 1265-6 (1991).

[13] K. Murata, H. Ushijima, H. Ueda, and K. Kawaguchi, J. Chem. Soc., Chem. Commun. pp. 567-569 (1992).

[14] S. Mizogami, M. Mizutani, M. Fukuda, and K. Kawabata, Synth. Met. 43, 3271 (1991).

[15] Y. Kopelevich, P. Esquinazi, J. Torres, and S. Moehlecke, J. Low Temp. Phys. 119, 691 (2000).

[16] P. Esquinazi, A. Setzer, R. Höhne, C. Semmelhack, Y. Kopelevich, D. Spemann, T. Butz, B. Kohlstrunk, and M. Lösche, Phys. Rev. B 66, 024429 (2002).

[17] P. Esquinazi, D. Spemann, R. Höhne, A. Setzer, K.-H. Han, and T. Butz, Phys. Rev. Lett. 91, 227201 (2003).

[18] P. Esquinazi, R. Höhne, K.-H. Han, D. Spemann, A. Setzer, M. Diaconu, H. Schmidt, and T. Butz, Induced magnetic order by ion irradiation of carbon-based structures (Elsevier Science, 2006), chap. 19, pp. 437-462, CarbonBased Magnetism, T. Makarova and F. Palacio (Eds.).

[19] H. Ohldag, T. Tyliszczak, R. Höhne, D. Spemann, P. Esquinazi, M. Ungureanu, and T. Butz, Phys. Rev. Lett. 98, 187204 (2007).

[20] J. Barzola-Quiquia, P. Esquinazi, M. Rothermel, D. Spemann, A. Setzer, and T. Butz, Nuclear Instruments and Methods in Physics Research B 256, 412 (2007).

[21] J. Barzola-Quiquia, P. Esquinazi, M. Rothermel, D. Spemann, T. Butz, and N. García, Phys. Rev. B 76, 161403 (2007).

[22] J. Coey, M. Venkatesan, C. Fitzgerald, A. Douvalis, and I. Sanders, Nature 420, 156 (2002).

[23] H.-C. Mertins, S. Valencia, W. Gudat, P. M. Oppeneer, O. Zaharko, and H. Grimmer, Europhys. Lett. 66, 743 (2004).

[24] J. F. Ziegler, The stopping and range of ions in matter (Pergamon Press, New York, 1977-1985).

[25] L. Pisani, B. Montanari, and N. Harrison, arXiv:0710.0957

[26] K. Schindler, N. García, P. Esquinazi, and H. Ohldag, unpublished.

* Electronic address: esquin@physik.uni-leipzig.de 